\title{
Polarization transfer in a spin-exchange optical-pumping experiment
}

\author{
Jyrki Rantaharju, ${ }^{*}$ Matti Hanni, ${ }^{\dagger}$ and Juha Vaara $\odot^{\ddagger}$ \\ NMR Research Unit, University of Oulu, P.O. Box 3000, FI-90014 Oulu, Finland
}

(Received 30 June 2020; accepted 26 August 2020; published 14 September 2020)

\begin{abstract}
Spin-exchange optical pumping (SEOP) enables hyperpolarization of magnetic noble gas nuclei and allows enormously enhanced signal in nuclear magnetic resonance studies in materials, biosciences, and medicine. We model the dynamics of the SEOP process taking place in a $\mathrm{Rb}^{129}{ }^{129} \mathrm{Xe}$ gas mixture and shed light on how the different microscopic processes influence the macroscopic polarization transfer. For each $\mathrm{Rb}-\mathrm{Xe}$ collision taking place in simulated molecular dynamics trajectory, we sample a time series of quantum-chemically preparametrized Hamiltonians. Combined electron and nuclear spin dynamics of each event is propagated by solving the corresponding Liouville-von Neumann equation. The rarely occurring, long-lived van der Waals molecules are seen to give the most significant contribution to polarization transfer under the simulated conditions ( $T=300 \mathrm{~K}, p=2.4 \mathrm{bar}$ ), in agreement with earlier findings. Besides the lifetime of the collision complex, the average and minimum $\mathrm{Rb}-\mathrm{Xe}$ interatomic distances characterize the efficiency of the polarization transfer events. We obtain insight into magnetization transfer in both individual binary collisions and van der Waals complexes and demonstrate a stepwise buildup of ${ }^{129} \mathrm{Xe}$ spin polarization upon bond-length oscillations in the latter.
\end{abstract}

DOI: 10.1103/PhysRevA.102.032813

\section{INTRODUCTION}

Nuclear magnetic resonance (NMR) is among the most influential spectroscopies [1]. However, NMR suffers from insensitivity due to the magnitude of the thermal equilibrium spin polarization, governed by Boltzmann occupations of the nuclear spin states $[2,3]$. To overcome this, hyperpolarization techniques [4-7] have been developed to create by up to several orders of magnitude enhanced nuclear polarization. Spin-exchange optical pumping (SEOP) [7] is used to attain high polarization levels of noble gas $\left({ }^{3} \mathrm{He},{ }^{83} \mathrm{Kr},{ }^{129 / 131} \mathrm{Xe}\right)$ nuclei, in interaction with electronically open-shell alkali-metal atoms. In SEOP, the ${ }^{2} S$ electronic state of, e.g., atomic $\mathrm{Rb}$ is spin-polarized by irradiation with circularly polarized light. The polarization is conveyed to the nuclei of gaseous xenon129 in both short-duration, so-called binary Rb-Xe collisions [8] and long-lived van der Waals (VDW) complexes of the two atoms [9-11]. The transfer occurs via the hyperfine (HF) interaction between the unpaired electron of the $\mathrm{Rb}$ atom and the magnetic (spin quantum number $I=\frac{1}{2}$ ) ${ }^{129}$ Xe nucleus, in $\mathrm{Rb}-\mathrm{Xe}$ interaction events. Depending on the efficiency of such collisions, the very large degree of electron spin polarization (see, e.g., Ref. [12]) can be converted to nuclear polarization that greatly exceeds the level attainable by thermal polarization in standard NMR magnets $[10,13]$. Continuous-flow

\footnotetext{
*Present address: School of Chemistry, University of Southampton, Southampton SO17 1BJ, United Kingdom.

${ }^{\dagger}$ Present address: Medical Research Center and Research Unit for Medical Imaging, Physics and Technology, P.O. Box 8000, FI-90014 University of Oulu, Finland; Dept. of Diagnostic Radiology, Oulu University Hospital, P.O. Box 50, FI-90029 Oulu, Finland.

*juha.vaara@iki.fi
}

SEOP is powerful in producing large quantities of hyperpolarized noble gases [14].

A pertinent question concerns the roles played by the binary $\mathrm{Rb}-\mathrm{Xe}$ collisions and the long-lived VDW complexes in the polarization transfer $[9,15,16]$, as well as the significance of experimental conditions (temperature $T$, pressure $p$, and the composition of the gas mixture). In particular, it has been found [9] that the important role played by the VDW molecules in polarization transfer decreases upon increasing $p$ and that, at high pressures, the binary collisions emerge as dominant. Models for the dynamics of the SEOP process have been developed [9,11], while the microscopic details of the polarization transfer have not been simulated from first principles. Present techniques allow detailed investigation of such processes by combining molecular dynamics (MD) simulations of the atomic trajectories, quantum-chemical (QC) calculations to extract the instantaneous parameters of the relevant spin Hamiltonian $\hat{H}(t)$, and spin dynamics (SD) simulations of the spin system, driven by the time series of $\hat{H}$. This approach has been applied to the electron [17] and both nuclear and electron [18] spin relaxation in an aqueous solution of $\mathrm{Ni}^{2+}$ ion. In the present paper, we introduce a multiscale simulation procedure for the microscopic processes of SEOP [7,11,19], a hyperpolarization method that is in widespread materials science and clinical use [20,21].

\section{THEORY AND COMPUTATIONS}

\section{A. Molecular dynamics simulations}

The calculations of this paper were carried out at one temperature $T$ and one pressure $p$ only, to introduce the multiscale simulation methodology in the context of a single set of experimental parameters. In this initial report we also only simulate 
$\mathrm{Rb}$ and $\mathrm{Xe}$, and we omit the other gas components that are used in SEOP experiments [14]: He, the purpose of which is to enhance electron spin polarization by broadening the $\mathrm{Rb}$ absorption to better match the laser emission profile [22], and $\mathrm{N}_{2}$, used to facilitate radiation-free relaxation of the excited $\mathrm{Rb}$ state [7]. We focus here on the qualitative aspects of the spin transfer to ${ }^{129} \mathrm{Xe}$ nuclear spin from $\mathrm{Rb}$ electron spin, once polarization of the latter has been achieved. In future work we will also address the dependence of the process on the values of $p$ and $T$, as well as different gas composition. The influence of experimental conditions on spin transfer efficiency has been discussed, e.g., in Ref. [23].

For this work, a MD trajectory of a $\mathrm{Rb}$ atom in Xe gas consisting of $2196 \mathrm{Xe}$ atoms was produced corresponding to $T=300 \mathrm{~K}$ and $p=2.4 \mathrm{bar}$, using the NAMD software [24]. The $a b$ initio $\mathrm{Rb}-\mathrm{Xe}$ potential energy function of Ref. [12] was used. Events (labeled $\epsilon$ ) of interacting $\mathrm{Rb}$-Xe pairs were extracted from evenly spaced ( $\tau=50 \mathrm{fs}$ ) MD snapshots. Before the $N V E$ (constant particle number, volume, and energy) production run, the conditions were prepared with the NPT (constant particle number, pressure, and temperature) simulation for $1 \mathrm{~ns}$, followed by 1-ns-long thermalization with $N V E$. With a MD time step of $1 \mathrm{fs}$, the resulting production trajectory was $268 \mathrm{~ns}$ long, and with the time step between adjacent saved snapshots of $\tau=50 \mathrm{fs}$, the data constituted 5360000 snapshots. The MDanalysis package [25] was used to read the binary files into the PYTHON code employed, in turn, to perform the SD simulations, as well as to calculate the radial distribution function.

\section{B. Spin Hamiltonian}

For each extracted $\mathrm{Rb}-\mathrm{Xe}$ interaction event, a time series of spin Hamiltonians [26], $\left\{\hat{H}_{j}^{\epsilon}\right\}$ [Eq. (1) below], was constructed based on QC calculations using fully relativistic densityfunctional theory (DFT) $[27,28]$. To the spin Hamiltonian of the $\mathrm{Rb}-{ }^{129} \mathrm{Xe}$ pair,

$$
\hat{H}(t)=\mu_{\mathrm{B}} \hat{\boldsymbol{S}} \cdot \boldsymbol{g}(t) \cdot \boldsymbol{B}+\hat{\boldsymbol{S}} \cdot \boldsymbol{\varepsilon}(t) \cdot \boldsymbol{M}+\hat{\boldsymbol{S}} \cdot \boldsymbol{A}(t) \cdot \hat{\boldsymbol{I}},
$$

we include the HF coupling tensor $(\boldsymbol{A})$ connecting the unpaired $\mathrm{Rb}$ electron to the ${ }^{129} \mathrm{Xe}$ nucleus, the $g$ tensor $(\boldsymbol{g})$ parametrizing the Zeeman interaction of the unpaired electron with the external magnetic field $\boldsymbol{B}$, and the tensor $\boldsymbol{\varepsilon}$ arising from the the electron spin-rotation coupling. $\hat{\boldsymbol{S}}$ and $\hat{\boldsymbol{I}}$ are the spins of the $\mathrm{Rb}$ electron and ${ }^{129} \mathrm{Xe}$, respectively. $\boldsymbol{M}$ is the rotational angular momentum of the $\mathrm{Rb}-\mathrm{Xe}$ pair, and $\mu_{\mathrm{B}}$ is the Bohr magneton.

The electron spin resonance (EPR) $g$ and $\boldsymbol{A}$ tensors were calculated for the $\mathrm{Rb}$-Xe dimer as a function of the internuclear separation using four-component DFT in the matrix Dirac-Kohn-Sham framework using the RESPECT quantumchemical package [29]. The hybrid exchange-correlation functional PBE0 [30] was employed. The completenessoptimized 27s25p21d1f basis [12] was used for the largecomponent wave function, for both $\mathrm{Rb}$ and $\mathrm{Xe}$. A common gauge origin at the center of mass of the Rb-Xe complex was employed for the $g$ tensor. The obtained components of $g$ and $\boldsymbol{A}$ parallel $\left(g_{\|}\right.$and $\left.A_{\|}\right)$and perpendicular $\left(g_{\perp}\right.$ and $\left.A_{\perp}\right)$ to the intermolecular $\mathrm{Rb}$-Xe axis were fitted to the analytical form

$$
T(r)=c+\frac{b}{r^{p_{0}+p_{1} r+p_{2} r^{2}}},
$$

for use in the analysis of present MD trajectories. Numerical values of the fit parameters can be found in Table I. Whereas QC calculations were used to parametrize $\boldsymbol{g}$ and $\boldsymbol{A}$ as functions of the interatomic $\mathrm{Rb}-\mathrm{Xe}$ distance $\left(R_{\mathrm{RbXe}}\right)$, the electron spin-rotation coupling tensor $\boldsymbol{\varepsilon}$ was extracted from Curl's approximation [31]

$$
\sum_{k} \varepsilon_{i k}(t) \mathcal{I}_{k j}(t)=\hbar^{2}\left[g_{e} \delta_{i j}-g_{i j}(t)\right],
$$

where $\mathcal{I}$ is the moment of inertia tensor of the $\mathrm{Rb}-\mathrm{Xe}$ pair and $g_{e}=2.0023$ is the free-electron $g$ factor that contributes to the isotropic part of the $g$ tensor, the latter with components $g_{i j}$. The instantaneous EPR tensors occurring in the MD simulation were furnished for each $\mathrm{Rb}-\mathrm{Xe}$ pair with $\boldsymbol{g}$ and $\boldsymbol{A}$ fitted to the precalculated QC property curves as functions of internuclear $R_{\mathrm{RbXe}}$ distance.

The ${ }^{129} \mathrm{Xe}$ nuclear spin rotation and ${ }^{85 / 87} \mathrm{Rb} \mathrm{HF}$ interactions were omitted in this initial investigation. Their roles can be expected to be more significant in the ${ }^{129} \mathrm{Xe}$ spin and unpaired electron spin relaxation, respectively [10].

In Fig. 1, the parallel and perpendicular components of $\boldsymbol{A}\left({ }^{129} \mathrm{Xe}\right)$ and $\boldsymbol{g}$ are plotted, as well as the $\mathrm{Rb}-\mathrm{Xe}$ radial distribution function (RDF) obtained for the gas mixture, against $R_{\mathrm{RbXe}}$. The RDF begins to deviate from unity at around $9 \AA$, well above the distance range at which the magnetic interactions $(\boldsymbol{A}$ and $\boldsymbol{g})$ start to deviate from their infinite-separation limit. The forms of the curves of $g_{\|}, g_{\perp}, A_{\|}$, and $A_{\perp}$ reflect the nature of the underlying interactions. $g$ is seen to be very anisotropic, with $g_{\perp} \ll g_{\|} \approx g_{e}$. This is due to the fact that, at the nonrelativistic limit, the dominating second-order cross term of the spin-orbit interaction and the orbital Zeeman interaction with the external magnetic field $\boldsymbol{B}$ [26] causes a significant shift of the $g$ tensor from $g_{e}$ in the perpendicular component $g_{\perp}$. In contrast, the orbital Zeeman interaction is ineffective in coupling to the electronically excited states when the internuclear axis of a linear molecule such as $\mathrm{RbXe}$ coincides with the direction of $\boldsymbol{B}$ in the $g_{\|}$component. Minor contributions in our fully relativistic calculation are responsible for the slight deviation of $g_{\|}$from $g_{e}$.

The xenon HF coupling tensor is seen to be nearly isotropic, i.e., $A_{\|} \approx A_{\perp}$, throughout the $\mathrm{Rb}$-Xe distance range. This can be understood in the light of the dominant Fermi contact-type coupling mechanism, which is isotropic. Again, deviations from isotropy result from the fact that we pursue a four-component calculation using the full relativistic HF operator, of which (among others) the isotropic contact operator can be obtained only at the nonrelativistic limit [32].

We define each visit of a Xe atom inside the radius of $9 \AA$ around the $\mathrm{Rb}$ atom to constitute a single interaction event. The simulated distribution of the number of such events as a function of the event lifetime is presented in Fig. 2. The number of events decays exponentially, with most of them having a lifetime shorter than 25 ps. Long-lived complexes are rare in the present simulation conditions. 
TABLE I. $g$ and ${ }^{129}$ Xe hyperfine coupling (A) tensor fitting parameters in Eq. (2).

\begin{tabular}{lcccrr}
\hline \hline Parameter & $g_{\|}$ & $g_{\perp}$ & Parameter & $A_{\|}$ & $A_{\perp}$ \\
\hline$c$ & 2.00234796 & 2.00236291 & $c(\mathrm{MHz})$ & -0.713669632 & -0.636729696 \\
$b\left(10^{-4} \AA\right)$ & -0.0224685200 & -7.39090180 & $b(\mathrm{GHz})$ & -1.31261227 & -1.38023054 \\
$p_{0}$ & -8.05431782 & -6.02841485 & $p_{0}$ & 0.328979699 & 0.341536219 \\
$p_{1}\left(\AA^{-1}\right)$ & 1.31710347 & 1.09654497 & $p_{1}\left(\AA^{-1}\right)$ & -0.134632780 & -0.0882831678 \\
$p_{2}\left(10^{-2} \AA^{-2}\right)$ & -3.59795315 & -3.21478859 & $p_{2}\left(10^{-2} \AA^{-2}\right)$ & 6.90283249 & 6.45460395 \\
\hline \hline
\end{tabular}

\section{Spin dynamics}

The time series of spin Hamiltonians $\left\{\hat{H}_{j}^{\epsilon}\right\}$ was used in SD simulation of spin transfer from the unpaired electron of the $\mathrm{Rb}$ atom to the ${ }^{129} \mathrm{Xe}$ nucleus, in each $\mathrm{Rb}-\mathrm{Xe}$ interaction event. The polarization transfer was obtained from the SD trajectories generated with a program written in the PYTHON [33] language, taking advantage of several of the routines of the SPINDYNAMICA [34] software package. We simulated the time evolution of the combined electron-spin-nuclear-spin density operator separately for each event $\epsilon$. A superpropagator of the form $[17,18]$

$$
\hat{\hat{\mathcal{P}}}_{\epsilon}(n \tau)=e^{\hat{\hat{L}}_{n}^{\epsilon} \tau} e^{\hat{\hat{L}}_{n-1}^{\epsilon} \tau} \ldots e^{\hat{\hat{L}}_{1}^{\epsilon} \tau}
$$

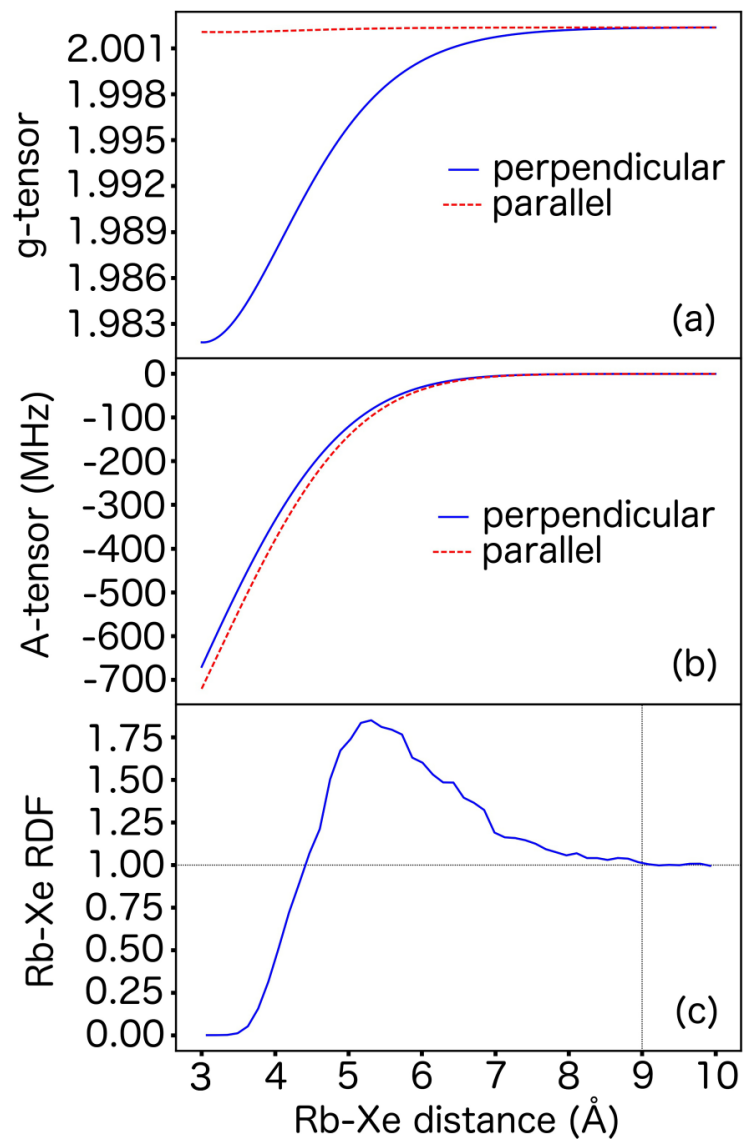

FIG. 1. Quantum-chemically computed components parallel to the internuclear axis and perpendicular to it of the (a) $g$ tensor and (b) ${ }^{129} \mathrm{Xe}$ hyperfine coupling tensor for the interacting $\mathrm{Rb}$-Xe dimer as functions of $R_{\mathrm{RbXe}}$. (c) The $\mathrm{Rb}-\mathrm{Xe}$ radial distribution function from molecular dynamics simulations. was constructed, where the Liouvillian superoperator $\hat{\hat{L}}_{j}^{\epsilon}$ relates to $\hat{H}_{j}^{\epsilon}$ through

$$
e^{\hat{L}_{j}^{\epsilon} \tau} \hat{\sigma}=e^{-i \hbar \hat{H}_{j}^{\epsilon} \tau} \hat{\sigma} e^{i \hbar \hat{H}_{j}^{\epsilon} \tau}
$$

The integer $n$ labels the SD time steps of length $\tau$. Hence, $\hat{P}_{\epsilon}$ is a propagator of the piecewise continuous solution to the pure-state Liouville-von Neumann equation. The SD of the observable state $\hat{O}$, traced from the initial state $\hat{S}_{z}$, is obtained as

$$
\mathscr{S}_{\hat{S}_{z}, \hat{O}^{\prime}}(n \tau)=\frac{\operatorname{Tr}\left[\hat{O} \hat{\hat{P}}_{\epsilon}(n \tau) \hat{S}_{z}\right]}{\sqrt{\operatorname{Tr}\left(\hat{O}^{2}\right) \operatorname{Tr}\left(\hat{S}_{z}^{2}\right)}} .
$$

This corresponds to an experimental setup where the unpaired electron of the $\mathrm{Rb}$ atom is assumed to be spin-polarized in the direction of the $z$ component of the Cartesian laboratory axis. The observable state $\hat{O}$ can be, e.g., any of the Cartesian components of the electron or nuclear spins (e.g., $\hat{I}_{x}, \hat{I}_{y}, \hat{I}_{z}$, or $\hat{S}_{z}$ ).

The events were divided into groups $D_{r}$ according to the lifetime of the Rb-Xe complex in question (see Fig. 3 below). Events in each $D_{r}$ approximate an ensemble, and within each range of lifetimes, the magnetization dynamics is obtained as a sum. We define the polarization transfer from state $\hat{S}_{z}$ to state $\hat{I}_{\alpha}(\alpha=x, y, z)$ as

$$
\mathscr{P}_{z, \alpha}^{r} \equiv \sum_{\epsilon \in D_{r}} \mathscr{S}_{\hat{S}_{z}, \hat{I}_{\alpha}}^{\epsilon}\left(n_{f}^{\epsilon} \tau\right)
$$

where $n_{f}^{\epsilon} \tau$ is the lifetime of the event $\epsilon$.

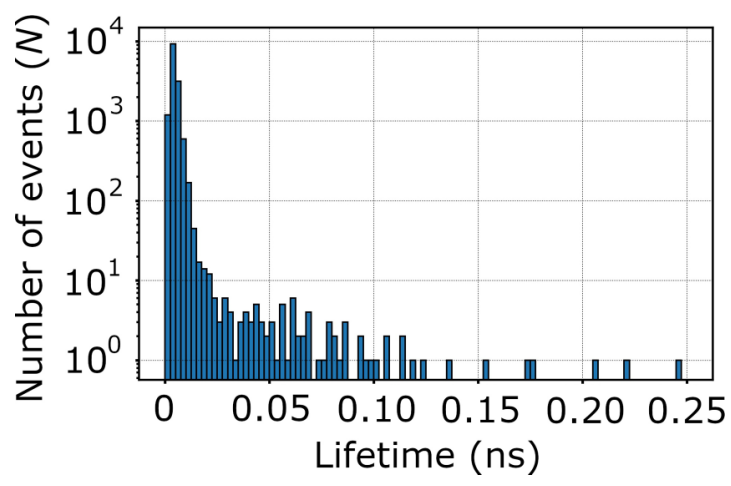

FIG. 2. Simulated distribution of the $\mathrm{Rb}-\mathrm{Xe}$ interaction events, $N$, as a function of their lifetime. 


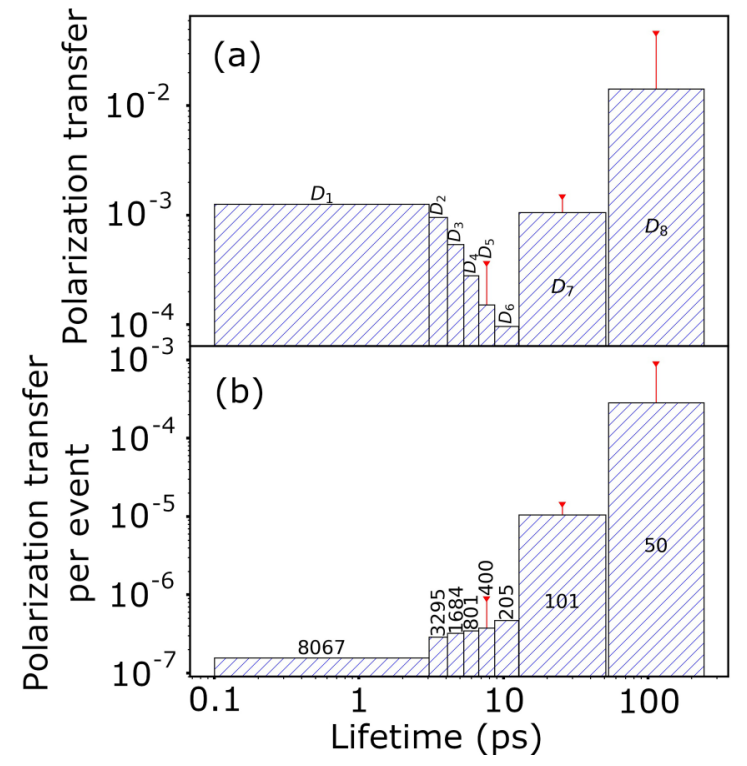

FIG. 3. (a) Distribution of polarization transfer, $\mathscr{P}_{z, z}^{r}$, in different-lifetime $\mathrm{Rb}$-Xe interaction events, at vanishing external magnetic field. Each bar represents a lifetime category $D_{1} \ldots D_{8}$ ranging from short, binary collisions to long-lived VDW complexes. For categories with significant statistical uncertainty, the upper half of the error bar is shown in red. (b) The polarization transfer of panel (a) divided by $N_{r}$, the number of events in $D_{r} . N_{r}$ is shown for each lifetime category in panel (b).

\section{RESULTS AND DISCUSSION}

\section{A. Polarization transfer and event lifetime}

Figure 3(a) shows a histogram of the polarization transfer from $\hat{S}_{z}$ to the observed state $\hat{I}_{z}$, in the chosen lifetime ranges $D_{r}$, with $r=1-8$, at $0 \mathrm{~T}$ external magnetic field strength. See Table II for tabulated numerical values. The specific ranges have been chosen to provide useful statistics corresponding to the number of events $N_{r}$ in each $D_{r}$. In the absence of an external magnetic field, the electronic Zeeman term of the Hamiltonian [Eq. (1)] is ineffective. The nature of the events ranges from hundreds-of-femtoseconds-long binary collisions to hundreds-of-picoseconds-long VDW molecules.

TABLE II. Simulated spin transfer $\mathscr{P}_{z, z}^{r}$ [Eq. (7)] and spin transfer per event $\mathscr{P}_{z, z}^{r} / N_{r}$ from the electron to ${ }^{129} \mathrm{Xe}$ nuclear spin in the different lifetime categories of Rb-Xe interaction events. $\tau=50 \mathrm{fs}$ is the time step used in the spin dynamics simulations.

\begin{tabular}{lcccc}
\hline \hline$D_{r}$ & Lifetime $n_{f}^{\epsilon}(\tau)$ & $N_{r}$ & $10^{2} \mathscr{P}_{z, z}^{r}$ & $10^{4} \mathscr{P}_{z, z}^{r} / N_{r}$ \\
\hline$D_{1}$ & $2<n_{f}^{\epsilon} \leqslant 61$ & 8067 & 0.12570 & 0.00156 \\
$D_{2}$ & $61<n_{f}^{\epsilon} \leqslant 82$ & 3295 & 0.09510 & 0.00289 \\
$D_{3}$ & $82<n_{f}^{\epsilon} \leqslant 106$ & 1684 & 0.05418 & 0.00322 \\
$D_{4}$ & $106<n_{f}^{\epsilon} \leqslant 135$ & 801 & 0.02790 & 0.00348 \\
$D_{5}$ & $135<n_{f}^{\epsilon} \leqslant 174$ & 400 & 0.01512 & 0.00378 \\
$D_{6}$ & $174<n_{f}^{\epsilon} \leqslant 256$ & 205 & 0.00967 & 0.00472 \\
$D_{7}$ & $256<n_{f}^{\epsilon} \leqslant 1069$ & 101 & 0.10577 & 0.10472 \\
$D_{8}$ & $1069<n_{f}^{\epsilon} \leqslant 4876$ & 50 & 1.41616 & 2.83232 \\
Total $\Sigma_{r}$ & & 14603 & 1.84960 & \\
\hline \hline
\end{tabular}

The proportion of binary collisions within the events of $D_{r}$ decreases with increasing $r$. The lifetime range $D_{8}$ consists solely of the Rb-Xe VDW complexes, whereas $D_{1}$ contains mostly short-lived, binary collisions.

The long-lived VDW molecules contained in the lifetime range $D_{8}$ give clearly the most significant contribution to the total polarization transfer under the present simulation conditions. The short collisions in $D_{1}$ give, despite their overwhelming abundance (Fig. 2), a smaller contribution by a factor of about 10 . The fact that binary collisions have only a little contribution to the total rate under the simulation conditions corresponding to high pressure, 2.4 bar or 1800 Torr and (in the present subsection) vanishingly small external magnetic field, is in line with experimental findings tabulated in Ref. [16]. In Fig. 3(b), the polarization transfers of different lifetime categories are scaled by the multiplicative factor $1 / N_{r}$. This highlights the significance of individual events in different lifetime ranges. The long interaction time in VDW complexes is reflected in their contribution to the spin transfer. Experimentally the role of binary collisions in spin transfer is seen to be independent of $p$, whereas the polarization transfer via VDW molecules becomes less efficient with increasing $p$ $[9,15,16]$. The latter can be rationalized through the increasing frequency of three-body collisions and, hence, shorter lifetimes of VDW complexes, upon elevating the pressure. Not all three-body events lead to the breakup of the VDW molecule, however, as discussed below. To verify the experimental trends as functions of $p, T$, and gas composition, simulations at different conditions will be carried out in the future.

The influence of the electron spin rotation tensor $\boldsymbol{\varepsilon}$ on the magnitude of the polarization transfer from the $\mathrm{Rb}$ electron to the ${ }^{129} \mathrm{Xe}$ nucleus turned out to be insignificant in our simulations. Analogously to the case of the nuclear spin rotation interaction contributing to ${ }^{129} \mathrm{Xe}$ relaxation, electron spin rotation is expected mainly to affect the relaxation of the $\mathrm{Rb}$ electron spin [35]. The magnitude of the effect of turning on the electron spin rotation interaction is in the scale of $10^{-6}$ in the case of $D_{1}$ (compared to the overall $10^{-3}$ ), in the scale of $10^{-8}$ in the case of $D_{2}\left(10^{-3}\right)$, and $10^{-11}$ for the rest of the lifetime categories $\left(10^{-4} \ldots 10^{-2}\right)$.

Polarization transfer from the initial state $\hat{S}_{z}$ to the transverse components $\hat{I}_{x}$ and $\hat{I}_{y}$ of the xenon magnetization, $\mathscr{P}_{z, x}^{r}$ and $\mathscr{P}_{z, y}^{r}$, respectively, has magnitude smaller than that of $\mathscr{P}_{z, z}^{r}$ by a factor of around 1000 .

\section{B. Polarization transfer as a function of external magnetic field strength}

Figure 4(a) shows the simulated dependence of the polarization transfer on the external magnetic field. See Table III for tabulated numerical values. Starting from $B=0.1 \mathrm{~T}$, the electronic Zeeman term in the Hamiltonian becomes increasingly significant, with the result that the magnitude of the polarization transfer decreases. Reference [16] lists experimental spin-exchange cross sections corresponding, separately, to van der Waals complexes and binary collisions at $B$ strengths ranging from $2 \times 10^{-6} \mathrm{~T}$ (effectively zero) to 4.7 $\mathrm{T}$. The cross sections decrease with increasing external magnetic field, which corroborates our observation. It has been pointed out that spin transfer in particularly (but not 


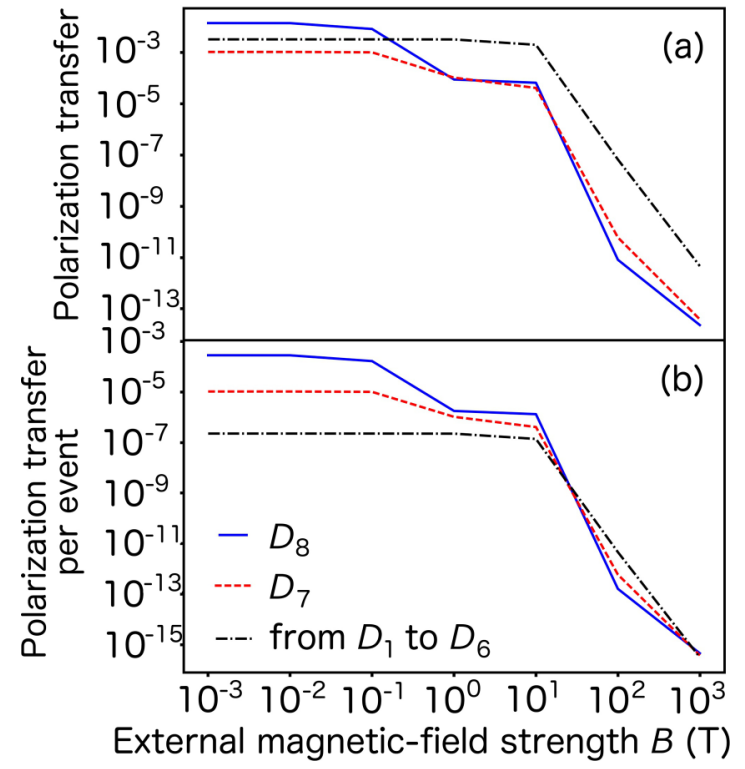

FIG. 4. (a) Simulated polarization transfer in the different event lifetime ranges (see Fig. 3) as a function of the external magnetic field strength. The contributions of the short lifetime ranges $D_{1} \ldots D_{6}$, are averaged over. (b) The same divided by the number of events $N_{r}$ within the individual lifetime categories.

only [16]) the VDW complexes becomes less efficient at high magnetic fields $[8,36]$. This trend is reproduced by our simulations.

We tested the length of the MD sampling interval in the context of finite $B$-field simulations, by recalculating the dependence of the polarization transfer on the external magnetic field strength with a SD time step doubled in length to $\tau=100 \mathrm{fs}$. With the tested magnitudes of $B$ in the range $10^{-3} \ldots 1 \mathrm{~T}$, the results obtained with the longer $\tau$ differ from those obtained with $\tau=50$ fs only by $0.01 \%$. The change at $10 \mathrm{~T}$ rises to $0.1 \%$, whereas $10 \%$ is obtained at $100 \mathrm{~T}$. This indicates that the sampling frequency is adequately small for our qualitative purposes.

\section{Polarization transfer and Rb-Xe distance}

Figure 5 illustrates the dependence of the polarization transfer on both the average and the minimum $\mathrm{Rb}-\mathrm{Xe}$ distance, $R_{\mathrm{RbXe}}$, occurring within the lifetime of the event (see

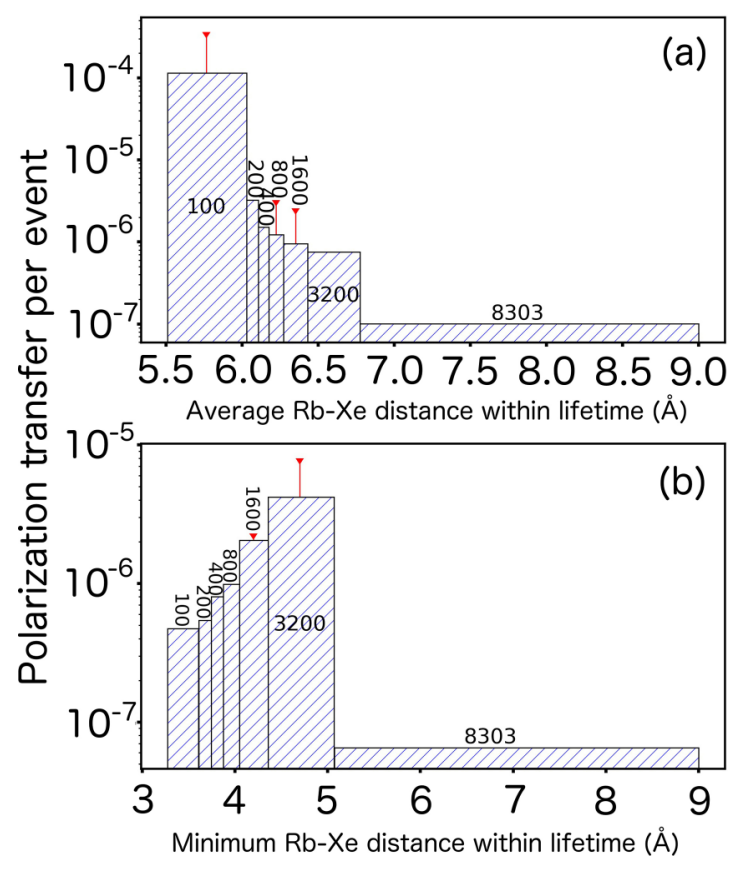

FIG. 5. (a) Distribution of polarization transfer in $\mathrm{Rb}-\mathrm{Xe}$ interaction events with different average $\mathrm{Rb}$-Xe distances, at vanishing external magnetic field. The average is calculated over the lifetime of the event. (b) The same as panel (a), but for different ranges of minimum $\mathrm{Rb}-\mathrm{Xe}$ distance occurring within the lifetime of the event. The number of events in each distance range is shown. For categories with significant statistical uncertainty, the upper half of the error bar is shown in red.

Tables IV and V for tabulated numerical values). The most significantly contributing lifetime range, $D_{8}$ in Fig. 3, consists solely of VDW molecules, and the large statistical error in $D_{8}$ illustrates the diversity among the complexes that occur in that lifetime range. Figure 5(a) shows that the average $R_{\mathrm{RbXe}}$ of some of the most significantly contributing events is between 5.5 and $6 \AA$, in the bar with 100 events. Based on the contribution to the polarization transfer, many of these events belong to $D_{8}$. On the other hand, Fig. 5(b) shows that the events with shortest minimum $R_{\mathrm{RbXe}}$ do not contribute significantly to the polarization transfer. Binary collisions are more prevalent among the events with the shortest minimum $R_{\mathrm{RbXe}}$.

TABLE III. Simulated polarization transfer as a function of the external magnetic field strength $B$ presented in Fig. 4 . The lifetime categories $D_{1} \ldots D_{6}$ have been summed over and categories 7 and 8 are shown individually. The last three columns show the same data divided by the number of events in the categories.

\begin{tabular}{lccccc}
\hline \hline$B(\mathrm{~T})$ & $\Sigma_{r=1-6} \mathscr{P}_{z, z}^{r}$ & $\mathscr{P}_{z, z}^{7}$ & $\mathscr{P}_{z, z}^{8}$ & $\left(\Sigma_{r=1-6} \mathscr{P}_{z, z}^{r}\right) / N_{1-6}{ }^{\mathrm{a}}$ & $\mathscr{P}_{z, z}^{7} / N_{7}$ \\
\hline $10^{-3}$ & $3.28 \times 10^{-3}$ & $1.06 \times 10^{-3}$ & $1.42 \times 10^{-2}$ & $2.27 \times 10^{-7}$ & $1.05 \times 10^{-5}$ \\
$10^{-2}$ & $3.28 \times 10^{-3}$ & $1.06 \times 10^{-3}$ & $1.41 \times 10^{-2}$ & $2.27 \times 10^{-7}$ & $1.05 \times 10^{-5}$ \\
$10^{-1}$ & $3.27 \times 10^{-3}$ & $1.02 \times 10^{-3}$ & $8.33 \times 10^{-3}$ & $2.27 \times 10^{-7}$ & $1.01 \times 10^{-5}$ \\
$10^{0}$ & $3.25 \times 10^{-3}$ & $1.05 \times 10^{-4}$ & $8.89 \times 10^{-5}$ & $2.25 \times 10^{-7}$ & $1.67 \times 10^{-4}$ \\
$10^{1}$ & $2.01 \times 10^{-3}$ & $4.15 \times 10^{-5}$ & $6.62 \times 10^{-5}$ & $1.39 \times 10^{-7}$ & $4.11 \times 10^{-7}$ \\
$10^{2}$ & $6.70 \times 10^{-8}$ & $6.05 \times 10^{-11}$ & $8.16 \times 10^{-12}$ & $4.64 \times 10^{-12}$ & $5.99 \times 10^{-13}$ \\
$10^{3}$ & $4.64 \times 10^{-12}$ & $3.86 \times 10^{-14}$ & $2.31 \times 10^{-14}$ & $3.21 \times 10^{-16}$ & $3.82 \times 10^{-16}$ \\
\hline \hline
\end{tabular}

${ }^{\mathrm{a}} N_{1-6}=\Sigma_{r=1-6} N_{r}$, the sum of the number of events in lifetime categories 1-6. 
TABLE IV. Simulated polarization transfer per event, in average $\mathrm{Rb}$-Xe distance ranges, from Fig. 5(a).

\begin{tabular}{lcc}
\hline \hline $\begin{array}{l}\text { Number } \\
\text { of events }\end{array}$ & $\begin{array}{c}\text { Average } \\
\text { distance } d(\AA)\end{array}$ & $\begin{array}{c}\text { Polarization transfer } \\
\text { per event }\end{array}$ \\
\hline 100 & $5.51 \leqslant d<6.03$ & $1.15 \times 10^{-4}$ \\
200 & $6.03 \leqslant d<6.11$ & $3.25 \times 10^{-6}$ \\
400 & $6.11 \leqslant d<6.18$ & $1.51 \times 10^{-6}$ \\
800 & $6.18 \leqslant d<6.27$ & $1.22 \times 10^{-6}$ \\
1600 & $6.27 \leqslant d<6.43$ & $9.48 \times 10^{-7}$ \\
3200 & $6.43 \leqslant d<6.78$ & $7.53 \times 10^{-7}$ \\
8303 & $6.78 \leqslant d<9.00$ & $1.00 \times 10^{-7}$ \\
\hline \hline
\end{tabular}

\section{Individual binary collisions}

For more insight into the details of the polarization transfer, the time evolutions of the spin transfer, $\mathscr{S}_{\hat{S}_{z}, \hat{I}_{z}}$, of four different, individual binary collisions are plotted in Fig. 6. Referring to the lifetime categories introduced in Fig. 3, the short event in Fig. 6(a) belongs to $D_{1}$, while the much longer-lived events in Figs. 6(b) and 6(c) belong to $D_{4}$ and $D_{6}$, respectively. In the latter two events, $\mathrm{Rb}$-Xe distances much shorter than those in the first event are probed. The event in panel (c) is close to being twice as long as the event in panel (b), which results in a larger polarization transfer by one-third in the longer event. The fact that the spin transfer contribution of both events is nevertheless on the same scale, roughly $10^{-7}$, suggests that the magnitude of the transfer is strongly influenced by the minimum Rb-Xe distance during the event lifetime, which is similar in the two examples. Figure 6(d) displays a deep binary collision belonging to lifetime category $D_{1}$ and in which the minimum interatomic distance of $4 \AA$ is probed. While still larger polarization transfer is observed than in the events depicted in Figs. 6(b) and 6(c), even in such a head-on binary collision the magnitude of $\mathscr{P}_{z, z}^{r}$ remains orders of magnitude smaller than that in the VDW complexes (vide infra).

\section{E. Individual van der Waals complexes}

The spin transfer in two individual, long-lived VDW complexes is illustrated in Fig. 7. Figure 7(a) shows the spin transfer process in an "unperturbed," purely binary VDW complex of $\mathrm{Rb}$ and $\mathrm{Xe}$ atoms. The $\mathrm{Rb}-\mathrm{Xe}$ distance of the VDW molecule is seen to undergo regular oscillation. Figures 7(b) and 7(c) illustrate, in contrast, a case where other perturber Xe atoms visit the immediate neighborhood of the

TABLE V. Simulated polarization transfer per event, in minimum $\mathrm{Rb}-\mathrm{Xe}$ distance ranges, from Fig. 5(b).

\begin{tabular}{lcc}
\hline \hline $\begin{array}{l}\text { Number } \\
\text { of events }\end{array}$ & $\begin{array}{c}\text { Minimum } \\
\text { distance } d(\AA)\end{array}$ & $\begin{array}{c}\text { Polarization transfer } \\
\text { per event }\end{array}$ \\
\hline 100 & $3.28 \leqslant d<3.61$ & $4.71 \times 10^{-7}$ \\
200 & $3.61 \leqslant d<3.74$ & $5.41 \times 10^{-7}$ \\
400 & $3.74 \leqslant d<3.88$ & $8.01 \times 10^{-7}$ \\
800 & $3.88 \leqslant d<4.05$ & $9.88 \times 10^{-7}$ \\
1600 & $4.05 \leqslant d<4.36$ & $2.05 \times 10^{-6}$ \\
3200 & $4.36 \leqslant d<5.07$ & $4.19 \times 10^{-6}$ \\
8303 & $5.07 \leqslant d<9.00$ & $6.53 \times 10^{-8}$ \\
\hline \hline
\end{tabular}
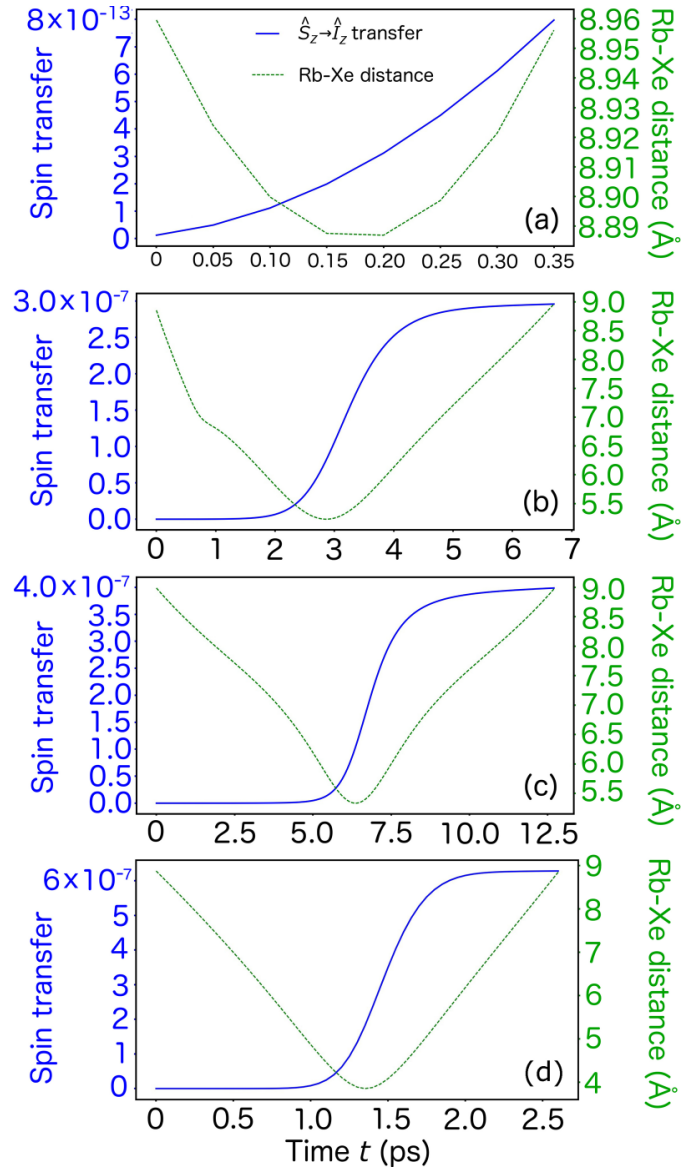

FIG. 6. Simulated polarization transfer between the unpaired $\mathrm{Rb}$ electron and ${ }^{129} \mathrm{Xe}$ nucleus and the $\mathrm{Rb}$-Xe distance in four binary collisions as a function of time. Events contained in the lifetime categories (a) $D_{1}$, (b) $D_{4}$, (c) $D_{6}$, and (d) $D_{1}$ (see Fig. 3).

long-lived complex during its lifetime. The lifetime of the complex depicted in Figs. 7(b) and 7(c) is the second-longest occurring in the entire MD trajectory. The Rb-perturber Xe distance of the disturbing three-body events, which influence both the molecular and spin dynamics of the VDW molecule, are plotted alongside the $\mathrm{Rb}-\mathrm{Xe}$ distance to the partner $\mathrm{Xe}$ atom in the complex. All the disturbing events occur as single collisions, and each of these events occurs with a different "intruder" xenon atom. Naturally, the intruder Xe of the first three-body event acts as the necessary third body for the formation of the VDW complex in the first place and, correspondingly, the last such event leads to the dissociation of the complex. The amplitude of the oscillation of the $\mathrm{Rb}-\mathrm{Xe}$ distance within the complex, as well as the minimum distance, is affected by the impact of the intruders. The minimum distance is reflected in $\mathscr{S}_{\hat{S}_{z}, \hat{I}_{z}}^{\epsilon}$, e.g., during the two intruder events at about 175 and 200 ps, between which the complex almost dissociates. This example shows that the intruders do not necessarily break up the VDW molecule, nor destroy the buildup of the ${ }^{129} \mathrm{Xe}$ magnetization (vide infra).

In both complexes depicted in Fig. 7, the spin transfer increases in a steplike manner whenever the $\mathrm{Rb}$-Xe distance goes through the minimum region of the periodic trajectory. There is a strong dependence of the Xe hyperfine coupling 


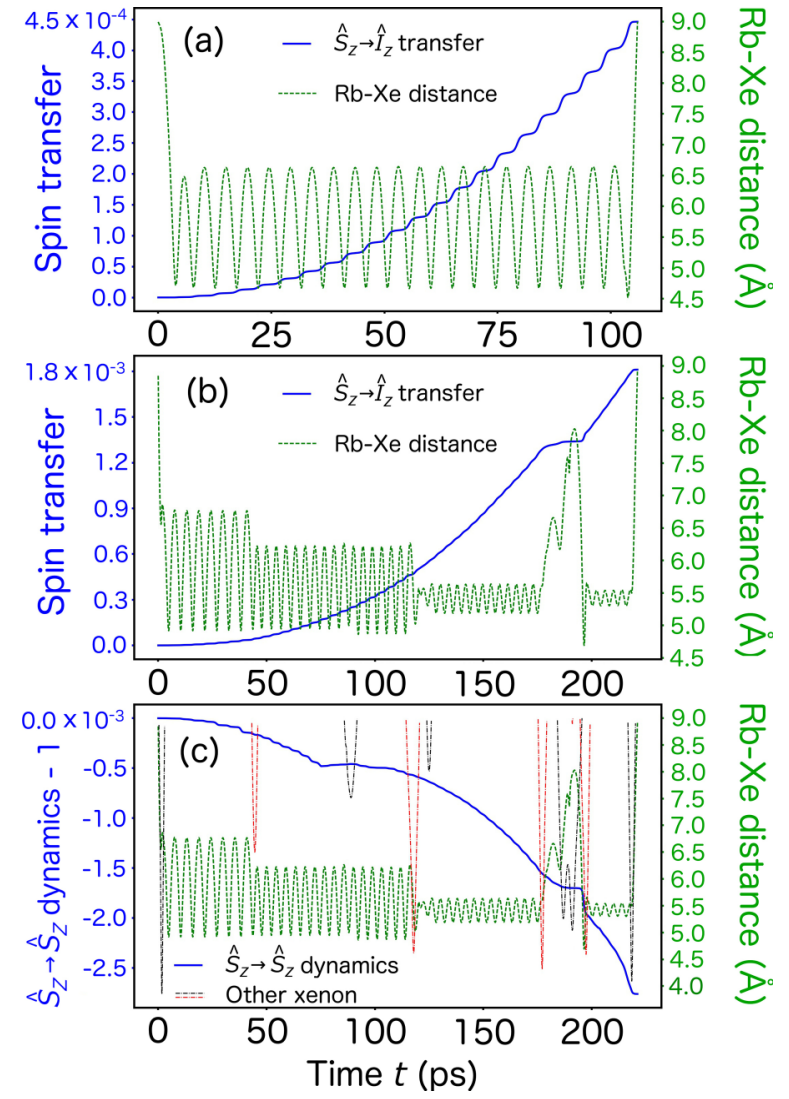

FIG. 7. Simulated polarization transfer between the unpaired Rb electron and ${ }^{129} \mathrm{Xe}$ nucleus and the $\mathrm{Rb}-\mathrm{Xe}$ distance, in two VDW complexes as a function of time [panels (a) and (b)]. Both molecules are contained in the $D_{8}$ lifetime category (see Fig. 3). (c) Simulated polarization decay of the unpaired electron $\left(\hat{S}_{z}\right)$ in the same VDW molecule as in panel (b). In addition, the Rb-Xe distances of all the collision events with other Xe atoms are plotted with dashed black and red lines.

interaction on the Rb-Xe distance (Fig. 1) and, hence, periodic modulation during the lifetime of the VDW complex, an effect speculated upon earlier [16,37]. In our simulations the oscillation period of the VDW complexes is circa $5 \mathrm{ps,}$ corresponding to $2 \times 10^{11} \mathrm{~Hz}$ frequency. In doing so, the magnitude of the additional spin transfer steps corresponding to one oscillation of the complex increases linearly. Figure 8 depicts how the magnitude of the steps in the spin transfer evolves during each oscillation cycle of the VDW complex. The minimum $\mathrm{Rb}-\mathrm{Xe}$ distance probed as a function of time is also plotted in both cases. In the case of the unperturbed complex [Fig. 8(a)], with its almost invariant oscillation cycles, the magnitude of the spin transfer steps is seen to increase linearly. This shows that the the magnitude of the increase is proportional to the number of previous steps. The propagator $\hat{P}_{\epsilon}$ accumulates with significant terms up to the second order in the time step $\tau$. The facts that the number of oscillations increases linearly with the lifetime of the complex and the magnitude of the spin transfer in an individual oscillation increases linearly with the number of oscillations accumulated so far, make up a quadratic total buildup of the polarization transfer as a function of time. In Fig. 8(b), the effect of the
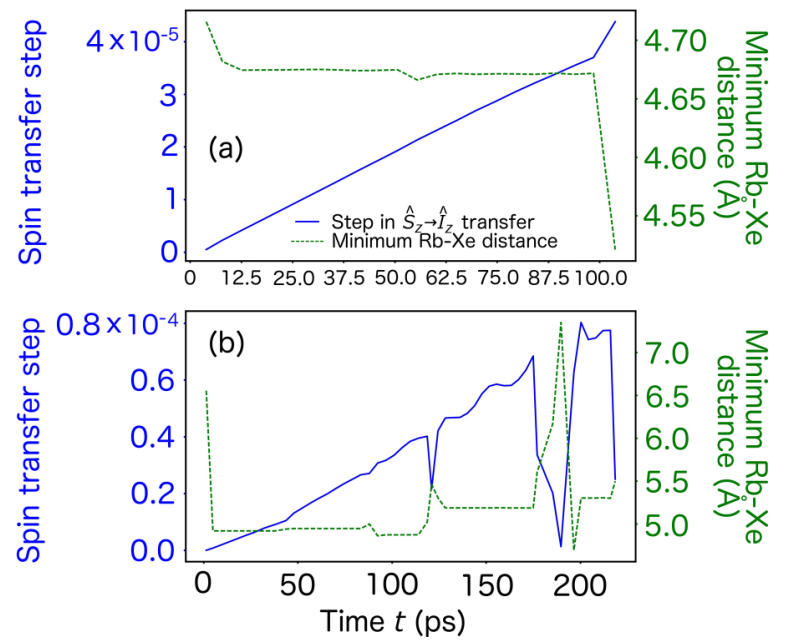

FIG. 8. Additional polarization transfer as a function of time between adjacent local maxima of the Rb-Xe distance in the van der Waals complexes depicted in Fig. 7. The minimum $\mathrm{Rb}-\mathrm{Xe}$ distance occurring in each oscillation is also illustrated. (a) Unperturbed complex. (b) Longer-lived, perturbed complex.

disturbing three-body events on the behavior is observed. It is noteworthy in Figs. 7(b) and 8(b) that the overall polarization transfer continues also after the third-body event that almost leads to a breakup of the complex at $175-200 \mathrm{ps}$.

Finally, Fig. 7(c) shows the decaying dynamics of the initial state $\hat{S}_{z}, \mathscr{S}_{\hat{S}_{z}, \hat{S}_{z}}^{\epsilon}$, reflecting loss of the electron spin polarization in the perturbed VDW complex of Figs. 7(b), 7(c), and 8(b). A three-dimensional animation of the entire event has been placed in the Supplemental Material [38].

\section{CONCLUSIONS}

Microscopic molecular and spin dynamics occurring in a $\mathrm{Rb}$-Xe spin-exchange optical-pumping experiment have been simulated to study the polarization transfer from the unpaired electron of the $\mathrm{Rb}$ atom to ${ }^{129} \mathrm{Xe}$ nuclei in the $\mathrm{Rb}$-Xe gas mixture. The study combined molecular dynamics simulation of the $\mathrm{Rb}$-Xe mixture, quantum-chemically preparametrized interactions in the spin Hamiltonian, and analysis of the MD trajectory to carry out a spin dynamics simulation of the combined electron and nuclear spin density as driven by the Hamiltonian. The investigation was performed as a function of the lifetime of the microscopic Rb-Xe interaction events occurring in the optical-pumping cell. The long-lived van der Waals molecules were shown to give the most significant contribution to the overall polarization transfer under the chosen simulation conditions. The short binary collision also gives an important, yet smaller contribution than the van der Waals complexes, due to the high number of the binary collision events. Experimental trends of polarization transfer as a function of the lifetime of the Rb-Xe interaction event as well as magnetic-field strength were reproduced by the simulation.

Examples of individual binary and van der Waals events could be scrutinized. A quadratic buildup of overall polarization transfer is seen in individual van der Waals molecules, with a steplike transfer increasing in magnitude for each bondlength oscillation of the complex. It is seen in an example 
van der Waals event that disturbing three-body incidents do not necessarily break up the complex, nor terminate the spin transfer process.

The present simulation was performed corresponding to only one set of experimental conditions: $T, p$, and the composition of the gas mixture. Further work will be needed to realistically address the influence of these factors at the microscopic level. However, this work suggests that the present type of multiscale simulations of the microscopic dynamics of the SEOP process can potentially aid in finding optimal experimental conditions, eventually to gain improved sensitivity in materials and biological NMR.

\section{ACKNOWLEDGMENTS}

The authors are grateful to Michal Repiský (Tromsø) for access to ReSpect software, as well as Pär Håkansson and Jiří Mareš (Oulu) for discussions. We thank the anonymous reviewer of our original manuscript for a number of useful and insightful comments. Financial support has been obtained from the University of Oulu (Exactus doctoral program, Kvantum Institute) and the Academy of Finland (Project No. 296292). Computational resources due to CSC (Espoo, Finland) and the Finnish Grid and Cloud Infrastructure project (persistent identifier urn:nbn:fi:research-infras-2016072533) were used.
[1] M. H. Levitt, Spin Dynamics: Basics of Nuclear Magnetic Resonance (Wiley, Chichester, 2001).

[2] L. Lumata, A. K. Jindal, M. E. Merritt, C. R. Malloy, A. D. Sherry, and Z. Kovacs, J. Am. Chem. Soc. 133, 8673 (2011).

[3] L. S. Lloyd, R. W. Adams, M. Bernstein, S. Coombes, S. B. Duckett, G. G. R. Green, R. J. Lewis, R. E. Mewis, and C. J. Sleigh, J. Am. Chem. Soc. 134, 12904 (2012).

[4] J. H. Ardenkjær-Larsen, B. Fridlund, A. Gram, G. Hansson, L. Hansson, M. H. Lerche, R. Servin, M. Thaning, and K. Golman, Proc. Natl. Acad. Sci. U.S.A. 100, 10158 (2003).

[5] R. W. Adams et al., Science 27, 1708 (2009).

[6] S. R. Bowers, in Encyclopedia of Nuclear Magnetic Resonance, edited by D. M. Grant and R. K. Harris (Wiley, Chichester, 2002), Vol. 9, p. 750.

[7] T. G. Walker and W. Happer, Rev. Mod. Phys. 69, 629 (1997).

[8] Y.-Y. Jau, N. N. Kuzma, and W. Happer, Phys. Rev. A 67, 022720 (2003).

[9] S. Appelt, A. B. Baranga, C. J. Erickson, M. V. Romalis, A. R. Young, and W. Happer, Phys. Rev. A 58, 1412 (1998).

[10] N. D. Bhaskar, W. Happer, and T. McClelland, Phys. Rev. Lett. 49, 25 (1982).

[11] W. Happer, E. Miron, S. Schaefer, D. Schreiber, W. A. van Wijngaarden, and X. Zeng, Phys. Rev. A 29, 3092 (1984).

[12] M. Hanni, P. Lantto, M. Repiský, J. Mareš, B. Saam, and J. Vaara, Phys. Rev. A 95, 032509 (2017).

[13] P. Nikolaou et al., Proc. Natl. Acad. Sci. U.S.A. 110, 14150 (2013).

[14] B. Driehuys, G. D. Cates, E. Miron, K. Sauer, D. K. Walter, and W. Happer, Appl. Phys. Lett. 69, 1688 (1996).

[15] G. D. Cates, R. J. Fitzgerald, A. S. Barton, P. Bogorad, M. Gatzke, N. R. Newbury, and B. Saam, Phys. Rev. A 45, 4631 (1992).

[16] C. V. Rice and D. Raftery, J. Chem. Phys. 117, 5632 (2002).

[17] J. Rantaharju, J. Mareš, and J. Vaara, J. Chem. Phys. 141, 014109 (2014).

[18] J. Rantaharju and J. Vaara, Phys. Rev. A 94, 043413 (2016).
[19] W. Happer, Rev. Mod. Phys. 44, 169 (1972).

[20] B. M. Goodson, J. Magn. Reson. 155, 157 (2002).

[21] S. J. Kruger, S. K. Nagle, M. J. Couch, Y. Ohno, M. Albert, and S. B. Fain, J. Magn. Reson. Imaging 43, 295 (2016).

[22] M. V. Romalis, E. Miron, and G. D. Cates, Phys. Rev. A 56, 4569 (1997).

[23] I. C. Ruset, S. Ketel, and F. W. Hersman, Phys. Rev. Lett. 96, 053002 (2006).

[24] J. C. Phillips et al., J. Comput. Chem. 26, 1781 (2005).

[25] N. Michaud-Agrawal, E. J. Denning, T. B. Woolf, and O. Beckstein, J. Comput. Chem. 32, 2319 (2011).

[26] J. E. Harriman, Theoretical Foundations of Electron Spin Resonance (Academic, New York, 1978).

[27] M. Repiský, S. Komorovský, E. Malkin, and O. L. Malkina, Chem. Phys. Lett. 488, 94 (2010).

[28] E. Malkin, M. Repiský, S. Komorovský, P. Mach, O. L. Malkina, and V. G. Malkin, J. Chem. Phys. 134, 044111 (2011).

[29] M. Repiský, S. Komorovský, V. G. Malkin, O. L. Malkina, M. Kaupp, and K. Ruud, with contributions from R. Bast, U. Ekström, S. Knecht, I. Malkin Ondik, and E. Malkin, ReSpect, relativistic spectroscopy DFT program, version 3.3.0, http://www.respectprogram.org.

[30] C. Adamo and V. Barone, J. Chem. Phys. 110, 6158 (1999).

[31] R. Curl, Jr., Mol. Phys. 9, 585 (1965).

[32] M. Reiher and A. Wolf, Relativistic Quantum Chemistry (WileyVCH, Weinheim, 2009).

[33] https://www.python.org/.

[34] C. Bengs and M. H. Levitt, Magn. Reson. Chem. 56, 374 (2018).

[35] T. G. Walker, Phys. Rev. A 40, 4959 (1989).

[36] Y.-Y. Jau, N. N. Kuzma, and W. Happer, Phys. Rev. A 66, 052710 (2002).

[37] F. A. Franz and C. Volk, Phys. Rev. A 18, 599 (1978).

[38] See Supplemental Material at http://link.aps.org/supplemental/ 10.1103/PhysRevA.102.032813 for animation of the MD in the perturbed VDW complex depicted in Figs. 7(b) and 7(c) (in a separate mp4 file). 